\title{
SINGULAR RIEMANNIAN METRICS, SUB-RIGIDITY VERSUS RIGIDITY
}

\author{
Samir BekKara And Abdelghani ZeghiB
}

\begin{abstract}
We analyze sub-Riemannian and lightlike metrics from the point of view of their rigidity as geometric structures. Following Cartan's and Gromov's formal definitions, they are never rigid, yet, in generic cases, they naturally give rise to rigid geometric structures!
\end{abstract}

\section{Contents}

1. On sub-Riemannian metrics

2. Lightlike metrics

3. Sub-rigidity of geometric structures, results

4. Proof of Theorem 3.2

5. On the proof of Theorem 3.1

5.2. The general case, generalized conformal structures

6.1. Gromov representation theorem for rigid structures 1210

6.2. No Gromov representation for sub-rigid structures 1211 Acknowledgments

\section{On sub-Riemannian metrics}

The following are variations on the concept of rigidity of geometric structures in a somehow "paradoxical" situation:

Sub-Riemannian metrics. A sub-Riemannian structure $(M, D, h)$ consists in giving on a manifold $M$ a hyperplane field $D \subset T M$ together with a metric $h$ defined on $D$ (and thought of as infinite on $T M-D$ ). An isometry of $h$ is a diffeomorphism preserving the structure.

The hyperplane field may be defined locally as the kernel of a 1-form $\omega_{0}$. There is however no canonical choice, any form $\omega=f \omega_{0}$ defines the same hyperplane.

Integrable case. If $D$ is integrable, say it defines a foliation $\mathcal{F}$, then $h$ is nothing but a leafwise Riemannian metric. We have for instance the particular global product case: $M=N \times S$, where the leaves $N \times\{*\}$ are endowed with a same metric $h_{0}$. Any family $\left(f_{s}\right)_{s \in S}$ in Iso $\left(N, h_{0}\right)$ determines an isometry of $(M, D, h)$.

\footnotetext{
Received by the editors 12 January, 2012.
} 
1.0.1. A correspondence: contact sub-Riemannian $\rightarrow$ Riemannian. We will henceforth assume that $D$ is a contact hyperplane, i.e., $\omega_{0} \wedge\left(d \omega_{0}\right)^{d}$ is a volume form where $\operatorname{dim} M=n=2 d+1$. Let us recall the (classical) construction of a natural Riemannian metric $\bar{h}$ associated to $(D, h)$. Observe that $d \omega_{0}$ is a symplectic form on $D$ and that for a function $f$, we have the equality $d\left(f \omega_{0}\right)=f d \omega_{0}$ (on $D$ ). Assume $D$ orientable, and let $\alpha$ be the Riemannian volume form derived from $h$ on it. Writing that $d \omega^{d}=\alpha$ on $D$ determines uniquely $f$, in other words the Riemannian metric (together with an orientation) allows one to choose a canonical contact form $\omega$. Recall that the Reeb field of $\omega$ is (uniquely) defined by $i_{R} d \omega=0$, and $\omega(R)=1$. Now, we extend the metric $h$ to a metric $\bar{h}$ on $T M$ by declaring that $R$ is unit and orthogonal to $D$. The orientation is actually irrelevant since the inverse orientation gives the same metric.

Isometry groups of Lie type. Summarizing up, a contact sub-Riemannian metric generates a Riemannian metric. In particular the isometry group $\operatorname{Iso}(M, h)$ is a (closed) sub-group of Iso $(M, \bar{h})$. Similarly for pseudo-groups of local isometries (i.e., isometries defined between open sets of $M$, and composed when this is possible). It then follows that the isometry group of $(M, h)$ as well as its local isometry pseudo-group are of Lie type (of finite dimension).

Cartan's finite-type condition. (see [18]) Let $H$ be a subgroup of $\mathrm{GL}_{n}(\mathbb{R})$. An $H$ structure on a manifold $M^{n}$ is a reduction of the structural group of its frame bundle $\mathrm{GL}^{(1)}(M)=P(M)$ to $H$. Equivalently, this is a section of $P(M) / H$ (assume here to simplify that $H$ is closed). A Riemannian metric corresponds to a $O(n)$-structure. A sub-Riemannian metric corresponds to an $H$ structure where $H$ is the subgroup of $\mathrm{GL}_{n}(\mathbb{R})$ preserving $\mathbb{R}^{n-1}$ and the standard Euclidean product on it. Its elements have the form:

$$
\left(\begin{array}{cc}
A & \vec{u} \\
0 & b
\end{array}\right)
$$

where $A \in O(n-1), \vec{u} \in \mathbb{R}^{n-1}$ and $b \in \mathbb{R}$.

Following Cartan, one associates to $H$ its $k$-prolongation $\mathcal{H}_{k}$, a space of symmetric $(k+1)$-multi-linear forms on $\mathbb{R}^{n}$ with values in $\mathbb{R}^{n}$. If $A \in \mathcal{H}_{k}$, and $v_{1}, \ldots, v_{k} \in \mathbb{R}^{n}$ are fixed, then $v \rightarrow A\left(v, v_{1}, \ldots, v_{k}\right)$ belongs to $\operatorname{End}\left(\mathbb{R}^{n}\right)$. By definition of a prolongation, the last element is assumed to be in the Lie algebra of $H$.

An $H$-structure has a finite type $k \in \mathbb{N}$, if $\mathcal{H}_{k}=0$. The principal result of the Cartan's theory is that the isometry group of an $H$-structure of finite type is a Lie group. The remarkable fact here is that being of finite type depends only on $H$ (as a subgroup of $\mathrm{GL}_{n}(\mathbb{R})$ ) and not on the structure itself. As an example, a subRiemannian metric has infinite type, no matter it is integrable or contact! If fact, the test of finiteness of type concerns the case of the flat translation-invariant $H$-structure on $\mathbb{R}^{n}$. The flow of a vector field $V$ preserves this structure iff, for any $x \in \mathbb{R}^{n}$, the derivative $D_{x} V$ belongs to the Lie algebra $\mathcal{H}$. The $(k+1)$-coefficient in the Taylor development in a linear coordinates of $V$ belongs to $\mathcal{H}_{k}$. Hence, finite type means $V$ is polynomial.

Gromov's RIGIDITY. (see $[2,3,8,11,16]$ ) Gromov's definition of geometric structures consists essentially in giving up the "infinitesimal homogeneity" in the case of Cartan's $H$-structures. As examples, functions and vector fields are geometric structures, and also is any "finite union" of geometric structures. Isometries are defined 
naturally. Gromov introduces a rigidity condition, which coincides with finiteness of type in the case of $H$-structures.

Rough-definition. If $\sigma$ is such a structure on a manifold $M$, and $x \in M$, let $\operatorname{Iso}_{x}^{L o c}(\sigma)$ be the group of (germs) of isometries defined in a neighborhood of $x$ and fixing $x$. For an integer $k$, denote by $\operatorname{Diff}_{x}^{k}(M)$ the group of $k$-jets of diffeomorphisms of $M$ fixing $x$. We have a map jet ${ }_{x}^{k}: \operatorname{Iso}_{x}^{\text {Loc }}(\sigma) \rightarrow \operatorname{Diff}_{x}^{k}(M)$. The intuitive idea of rigidity (of order $k$ ) is that jet ${ }_{x}^{k}$ is injective: an isometry is fully determined by its $k$-jet. We say in this case that $\sigma$ is Iso-rigid at order $k$. For example, a Riemannian metric is Iso-rigid at order 1: an isometry is determined by giving its derivative at some point. In the case of sub-Riemannian metrics, jet $_{x}^{1}$ is injective in the contact case (since it generates a Riemannian metric), but for no $k$, jet ${ }_{x}^{k}$ is injective in the integrable one.

Definition. We then conclude a divergence between this intuitive formalization of rigidity and Cartan's finiteness of type. The true Gromov's definition is actually of infinitesimal nature. For an $H$-structure $\sigma$, one defines the group $\operatorname{Iso}_{x}^{k+1}(\sigma) \subset$ $\operatorname{Diff}_{x}^{k+1}(M)$ as the group of $(k+1)$-jets of diffeomorphisms preserving $\sigma$ up to order $k$ at $x$. For example, if $\sigma$ is a Riemannian metric, then a (local) diffeomorphism $f$ gives rise to a $(k+1)$-infinitesimal isometry belonging to Iso ${ }_{x}^{k+1}(\sigma)$ if $f^{*} \sigma-\sigma$ vanishes up to order $k$ at $x$. (In the general case of a geometric structure $\sigma$ of order $r, f$ is a $(k+r)$-isometry if $f^{*} \sigma$ and $\sigma$ have the same $k$-jet at $\left.x\right)$. The true definition of $k$-rigidity is that jet ${ }^{k}: \mathrm{Iso}_{x}^{k+1}(\sigma) \rightarrow \mathrm{Iso}_{x}^{k}(\sigma)$ is injective for any $x$.

Example. Let us see how this injectivity default happens in the example of the contact form $\omega=d z+x d y-y d x$ on $\mathbb{R}^{3}$, endowed with the restriction of $d x^{2}+d y^{2}$. It corresponds to a left invariant contact sub-Riemannian structure on the Heisenberg group, and hence it is homogeneous. Consider $f:(x, y, z) \rightarrow(x+\delta(z), y+\delta(z), z)$. Assume $\delta(0)=0$, then, $f(0)=0$. Thus, jet ${ }_{0}^{k+1} f$ determines a $(k+1)$-isometry at 0 , iff, $\frac{\partial^{k} \delta}{\partial z^{k}}(0)=0$ or equivalently jet ${ }_{0}^{k}(f)=1$ (that is $f$ has the same jet as the identity). So any such $\delta$ with $\frac{\partial^{k+1} \delta}{\partial z^{k+1}}(0) \neq 0$ determines an isometry violating the injectivity of $\mathrm{IsO}_{0}^{k+1} \rightarrow \mathrm{IsO}_{0}^{k}$.

Some remarks. (1) Gromov's definition strictly coincides with finiteness of type in the case of $H$-structures (see [2], Example 3.17), and thus sub-Riemannian metrics are $k$-rigid for no $k$.

(2) Between Cartan and Gromov, there have been many substantial contributions on this subject e.g., by S. Kobayaschi, V. Guillemin, S. Sternberg, I. Singer...

(3) Let us end this criticism on the rough definition of Iso-rigidity by noting that a generic geometric structure (e.g., a Riemannian metric) has no non-trivial local isometries, in which case the local rigidity condition is empty. In contrast, it is the infinitesimal rigidity condition (even empty) that allows one to associate "rigid" (solid!) objects to $\sigma$, independently of the fact that it has or not local isometries. Indeed, it is proved in both Cartan and Gromov situations, that $k$-rigidity (or $k$ finiteness of type) allows one to construct a parallelism canonically associated to $\sigma$ defined on the $k$-frame bundle $\mathrm{GL}^{(k)}(M) \rightarrow M$ (this is the usual frame bundle for $k=1$ ) (see for instance [2] and Theorem 2 in [8]). This produces in particular the 
Levi-Civita connection and hence geodesics for Riemannian metrics. Also, the Lie group property is proved by means of this framing. ${ }^{1}$

\section{Lightlike metrics}

Duality. Our original motivation was to study rigidity of lightlike metrics. They are simply $H$-structures where $H$ consists of matrices:

$$
\left(\begin{array}{cc}
b & \vec{u} \\
0 & A
\end{array}\right)
$$

where $A \in O(n-1), \vec{u} \in \mathbb{R}^{n-1}$ and $b \in \mathbb{R}$. Observe that this is exactly the dual of the group defining sub-Riemannian metrics, that is, the automorphism $A \in \mathrm{GL}_{n}(\mathbb{R}) \rightarrow$ $A^{*-1} \in \mathrm{GL}_{n}(\mathbb{R})$ sends one group onto the other.

More geometrically, one defines a lightlike scalar product on a vector space as a positive symmetric bilinear form having a kernel of dimension one. A lightlike metric on a manifold $M$ is a tensor, which is a lighlike scalar product on each tangent space. More generaly, a lighlike metric on a vector bundle $E \rightarrow M$ consists in giving a onedimensionnal sub-bundle $N \subset E$ together with a Riemannian metric on $E / N$. If one defines a sub-Riemannian metric on $E \rightarrow M$ as a codimension 1 sub-bundle $D \subset E$ endowed with a Riemannian metric, then one gets a duality:

lightlike metric on $E \leftrightarrow$ sub-Riemannian metric on (the dual) $E^{*}$.

In other words, a lightlike metric $g$ on a manifold $M$ consists in giving a line subbundle (direction field) $N \subset T M$, and a Riemannian metric on $T M / N$. The direction field $N$ and the one-dimensional foliation $\mathcal{N}$ that it generates are called characteristic.

Natural situations. Lightlike metrics appear naturally as induced metrics on submanifolds of Lorentz manifolds. Indeed let $(L, h)$ be a Lorentz manifold, and $M \subset L$ a submanifold such that for any $x \in M$, the restriction $h_{x}$ on $T_{x} M$ is degenerate. Then, this is a lightlike metric on $M$, i.e., $h_{x}$ has a kernel of dimension 1 and is positive on $T_{x} M$. As an example, by definition, characteristic hypersurfaces of the D'Alembertian operator on $L$ are lightlike hypersurfaces. Also, horizons (in particular of black holes if any) of subsets of $L$ are topological hypersurfaces and are lightlike when they are smooth.

Now we give two opposite classes of examples of lightlike metrics which correspond, by duality to the integrable and contact cases of the sub-Riemannian situation, respectively:

Transversally Riemmannian lightlike metrics. A lightlike metric on a manifold $I$ of dimension 1 is just 0 . Consider now a direct product of $(I, 0)$ with a Riemannian metric $(Q, h)$. This gives a lightlike metric $h \oplus 0$ on $Q \times I$. A lightlike metric $g$ on a manifold $M$ is called transversally Riemannian if it is locally isometric to such a (direct) product. This is equivalent to the fact that the flow of any vector field tangent to the characteristic direction $N$ preserves $g$ (it suffices that this happens for one non-singular such vector field).

\footnotetext{
${ }^{1}$ At a more concrete level, one observes that for a Riemannian metric, if $\nabla^{1}$ and $\nabla^{2}$ are two connections satisfying the Levi-Civita requirements, then $S=\nabla^{1}-\nabla^{2}: T M \times T M \rightarrow T M$ defines at any $x \in M$ a 2-prolongation of the orthogonal group $O(n)$, and hence vanishes. Thus 1-rigidity and existence of the Levi-Civita connection have the same essence.
} 
Generic lightlike metrics. Let $X$ be a vector field tangent to $N$. One sees that $N$ annihilates the Lie derivative $L_{X} g$ (i.e., $L_{X} g(u, v)=0$, if $u \in N$ ). Furthermore, $L_{X} g$ is conformally well defined: if $X^{\prime}$ is another vector field tangent to $N$, then $L_{X^{\prime}} g=f L_{X} g$, for some function $f$ on $M$. We say that $g$ is generic if $L_{X} g$ has maximal rank, i.e., its kernel is exactly $N$. This therefore defines a conformal pseudoRiemannian structure on $T M / N$.

Rigidity flavors. Exactly as in the sub-Riemannian case, lightlike metrics have infinite type and thus are not rigid. Indeed, the local isometry group has infinite dimension for any transversally Riemannian metric. For example, if $M=\mathbb{R}^{n-1} \times \mathbb{R}$, with the metric $d x_{1}^{2}+\ldots+d x_{n-1}^{2}$, then any map $f(x, t)=(x, l(x, t))$ is isometric.

The key observation of [5] was a kind of Liouville theorem for the lightcone $C o^{n}$, $n \geq 4$. This is $\mathbb{R}^{+} \times S^{n-1}$ endowed with the lightlike metric $g_{(t, x)}=e^{2 t} C a n_{x}$, where $C a n$ is the usual metric on $S^{n-1}$. This is in fact the lightcone at 0 in the Minkowski space $\mathrm{Min}^{n+1}$. The Lorentz group $\mathrm{O}^{+}(1, n)$ acts isometrically on $\mathrm{Min}^{n+1}$ and hence on $C o^{n}$. The observation is that any local isometry of $C o^{n}$ coincides with the restriction of an element of $O^{+}(1, n)$.

A correspondence: "generic transversally conformal Lightlike geometry" $\leftrightarrow$ Conformal Riemannian geometry. The cone situation generalizes to that of transversally conformal lightlike structure. This means that the flow of any $X$ tangent to $N$ is conformal, equivalently $L_{X} g=f g$ for some function $f$. Locally, $M=Q \times I$ where $I$ is an interval, and $g_{(q, r)}=c(q, r) h_{q}$, where $h$ is a Riemannian metric on $Q$.

Assume $\phi$ is an isometry of $(M, g)$, then it acts on $Q$, the quotient space of its characteristic foliation and induces a diffeomorphism $\psi$, which is obviously conformal for $(Q, h)$.

Conversely, let $\psi$ a conformal transformation of $(N, h)$, and let us look for an isometry of $(M, g)$ of the form $\phi:(q, r) \rightarrow(\phi(q), \delta(q, r))$. We assume here $g$ generic, which means that $\frac{\partial c(q, r)}{\partial r} \neq 0$. Let us assume that $I=\mathbb{R}$, and for any $q$, the map $r \rightarrow c(q, r)$ is a global diffeomorphism of $\mathbb{R}$. If $f$ is the conformal distortion of $\psi$, that is $\psi^{*} h=f h$, then $\phi$ is isometric iff $c(\phi(q), \delta(q, r)) f(q)=c(q, r)$. Our hypotheses imply that for any fixed $q, \delta(q, r)$ can be uniquely chosen, and hence a conformal transformation of $(N, h)$ admits a unique isometric lifting on $(M, g)$. (One may compare with a somehow similar construction in [13]).

\section{Sub-rigidity of geometric structures, results}

We have the following infinitesimal result for lightlike metrics (where $\operatorname{jet}_{x}^{k}(\phi)=1$, means that $\phi$ has the same $k$-jet as the identity at $x$ ):

Theorem 3.1. Let $g$ be a generic lightlike metric on a manifold $M$ of dimension $n \geq 4$. Then, a 3-infinitesimal isometry with a trivial 1-jet, has a trivial 2-jet:

$$
\phi \in \mathrm{Iso}_{x}^{3}, \operatorname{jet}_{x}^{1}(\phi)=1 \Longrightarrow \operatorname{jet}_{x}^{2}(\phi)=1
$$

This notion was actually brought out by Benveniste-Fisher in [4] under the name of almost-rigidity. We believe here that the word "sub-rigid" is more telling (see also $[12])$.

In order to keep an elementary level of exposition, we restrict ourselves to geometric structures of order 1 , that is, $\mathrm{GL}_{n}(\mathbb{R})$-equivariant maps $P(M)\left(=\mathrm{GL}^{(1)}(M)\right) \rightarrow Z$, 
where $P(M)$ is the frame bundle of $M$ and $Z$ is a manifold with a $\mathrm{GL}_{n}(\mathbb{R})$-action. The classical case of an $H$ structure corresponds to the homogeneous space $Z=$ $\mathrm{GL}_{n}(\mathbb{R}) / H$.

Definition 3.1. A geometric structure $\sigma$ is $(k+s, k)$-sub-rigid, if any $(k+s)$-isometry whose $k$-jet is trivial has a trivial $(k+1)$-jet; formally, if $\operatorname{Im}_{\mathrm{x}}^{\mathrm{k}+\mathrm{s}, \mathrm{k}+1}$ denotes the image of $\mathrm{Iso}_{x}^{k+s} \rightarrow \mathrm{Iso}_{x}^{k+1}$, then, for any $x, \mathrm{Im}_{\mathbf{x}}^{\mathrm{k}+\mathrm{s}, \mathrm{k}+1} \rightarrow \mathrm{Iso}_{\mathrm{x}}^{\mathrm{k}}$ is injective.

\section{Remarks 3.1.}

(1) $(k+1, k)$-sub-rigidity means $k$-rigidity.

(2) In particular, a $(k+s, k)$-sub-rigid structure is Iso-rigid at order $k$.

(3) The theorem above states that generic lightlike metrics are $(3,1)$-sub-rigid.

In the sub-Riemannian case, we have

Theorem 3.2. A contact sub-Riemannian metric is $(4,1)$-sub-rigid.

Example. The paradigmatic example of sub-rigid structures presented in [4] was that of a degenerate framing. That is, on $\mathbb{R}^{n}$, a system of vector fields $x \rightarrow\left(X_{1}(x), \ldots\right.$, $X_{n}(x)$ ), which are linearly independent everywhere except at an isolated point, say 0 . As an example, take $n=1$, and the geometric structure being a vector field $X(x)=f(x) \frac{\partial}{\partial x}$. A diffeomorphism $\phi$ is isometric if $\phi^{\prime}(x) f(x)=f(\phi(x))$. If $f$ does not vanish, then we have a true parallelism, and it is 0-rigid: trivial 0 -jet implies trivial 1-jet, say at the point $0 \in \mathbb{R}$; in other words, $\phi(0)=0$ implies $\phi^{\prime}(0)=1$.

Assume now that $f(0)=0$, then $\phi$ is isometric up to order $k+1$ at 0 , if it satisfies, at the point 0 , all the equations obtained by taking derivatives up to order $k$ of the equality: $\phi^{\prime}(x) f(x)=f(\phi(x))$. Assume $f$ has a zero of order $d$ at 0 , e.g. $f(x)=x^{d}$, and that jet $_{0}^{1}(\phi)=1$, i.e., $\phi(0)=0$ and $\phi^{\prime}(0)=1$; then we need derivatives of $\phi$ up to order $d+2$ in order to conclude that $\operatorname{jet}_{0}^{2}(\phi)=1$, i.e., $\phi^{\prime \prime}(0)=0$. Therefore, the structure is $(d+2,1)$-sub-rigid.

\section{Remarks 3.2.}

(1) An essentially equivalent example is given in ([11], Section 5.11.B) to show weakness of Iso-rigidity in comparison with rigidity.

(2) One may think following [4] that, as above, there is always a degeneracy phenomenon behind sub-rigidity. One may in particular ask if a sub-rigid structure is rigid on an open dense set? However, the examples of the lightlike structure on the Minkowski lightcone, and the standard contact sub-Riemannian metric on the Heisenberg group, show that sub-rigid structures can be homogeneous. They are in particular nowhere rigid.

\section{Proof of Theorem 3.2}

Proof. Let $(D, h)$ be a contact sub-Riemannian structure on $M$. For computation, it is useful to see $(D, h)$ as an equivalence class of pairs $(\omega, h)$ where $\omega$ is any contact form defining $D$. The correspondence $(\omega, h) \rightarrow \bar{h}$ discussed in Section 1.0.1 does not depend on the particular choice of $\omega$. We will show that the 1-jet of $\bar{h}$ is determined by the the 3 -jet of $(\omega, h)$. 
Let $X_{1}, \ldots, X_{2 d}$ be a local system of smooth vector fields generating $D$, where $\operatorname{dim} M=2 d+1$. The normalized form $\omega^{\prime}=f \omega$ (determined canonically by $(\omega, h)$, that is its maximal power coincides with the $h$-volume form on $D$ ) is defined by

$$
f d \omega^{d}\left(X_{1}, \ldots, X_{2 n}\right)=\operatorname{det}\left(h\left(X_{i}, X_{j}\right)_{i j}\right) .
$$

Its Reeb vector field $R$ is defined algebraically by

$$
d f\left(X_{i}\right) \omega(R)+f d \omega\left(X_{i}, R\right)=0, \text { and } f \omega(R)=1, \text { for all } i .
$$

If $X_{0}$ is a vector field transverse to $D$, then $\bar{h}\left(X_{i}, X_{0}\right)_{i j}, i, j \geq 0$, are given by the $h\left(X_{i}, X_{j}\right), i, j>0$, and the coordinates of $R$ in the moving frame $\left\{X_{0}, \ldots, X_{2 d}\right\}$. In particular the first derivatives of $\bar{h}$ come from third derivatives of $(\omega, h)$.

Let $f$ be a diffeomorphism, $f^{*} \omega=\omega_{1}$ and $f^{*} h=h_{1}$. Thus $f^{*} \bar{h}=\overline{h_{1}}$. If $f$ is a 4 -isometry for $(\omega, h)$, then by definition $\omega$ and $\omega_{1}$ (resp. $h$ and $h_{1}$ ) coincide up to order 3 . It then follows that $\bar{h}$ and $\overline{h_{1}}$ coincide up to order 1 , that is $f$ a 2 -isometry for $\bar{h}$. If $f$ was merely a 4 -isometry for $(D, h)$, then, $\omega$ will coincide up to order 3 with a multiple $g \omega_{1}$, which leads to the same conclusion for $\bar{h}$.

To prove $(4,2)$-sub-rigidity for $(D, h)$, apply the 1-rigidity (say the $(2,1)$-subrigidity) of Riemannian metrics. We get here that, if $f$ is a 4 -isometry with a trivial 1-jet, then it has a trivial 2-jet.

Example. Endow $\mathbb{R}^{3}$ with the contact hyperplane field determined by the form $\omega=$ $d z-x d y$ together the restriction of $d x^{2}+d y^{2}$ on it. The map $f(x, y, z)=(x+$ $\left.\frac{1}{2} z^{2}, y-\frac{1}{2} z x^{2}, z+\frac{1}{2} y z^{2}\right)$ belongs to Iso $_{0}^{3}$. It has a trivial 1 -jet, but a non-trivial 2 -jet. Therefore, the structure is not $(3,1)$-sub-rigid.

\section{On the proof of Theorem 3.1}

We give in what follows hints on the proof of Theorem 3.1. Details, especially for Sections 5.1.2 and 5.2 will appear in [6].

5.1. The transversally conformal case. Let us consider first the transversally conformal case. Locally, $M=Q \times I$, and $g_{(q, r)}=c(q, r) h_{q}$. An isometry $\phi$ has the form $\phi:(q, r) \rightarrow(\psi(q, r), \delta(q, r))$. Since $\phi$ preserves the characteristic foliation of $g$, it acts on the quotient space $Q$, that is, $\psi$ does not depend on $r$.

5.1.1. The local isometry equation. The isometric equation is:

$$
c(\psi(q), \delta(q, r)) \phi^{*} h=c(q, r) h
$$

That is, $\phi$ is conformal, say with a distortion $f$ (i.e., $\phi^{*} h=f h$ ), which satisfies the cocycle property: $c(\psi(q), \delta(q, r)) f(q)=c(q, r)$.

INFINITESIMAL CASE. For the sake of simplicity, even for infinitesimal isometries, we will assume that their $\psi$-part depends only on $q$ (what is a priori true in this case is that the derivatives on $\psi$ with respect to $r$ vanish according to the order of the infinitesimal isometry).

Fix a point, say $(q, r)=(0,0)$. The fact that $\phi=(\psi, \delta)$ is isometric of order 1 (and fixes $(0,0))$ means exactly that the previous equation is satisfied for $(0,0)$. So $\psi(0)=0, \delta(0,0)=0$, and $\psi$ is conformal at order 1 . 
First step: $\phi \in \operatorname{Iso}_{(0,0)(g)}^{3}$ and $\operatorname{jet}_{(0,0)}^{1} \phi=1 \Longrightarrow \operatorname{jet}_{0}^{3} \psi=1$ :

- The fact that $\phi$ has a trivial 1-jet translates to: $D_{0} \phi=1, \frac{\partial \delta}{\partial q}=0$ and $\frac{\partial \delta}{\partial r}=1$.

- The fact that $\phi \in \mathrm{Iso}_{(0,0)}^{2}(g)$ means that the second derivatives of $\phi$ at $(0,0)$ satisfy all the equalities obtained by derivating the previous equation. Here, using that $\frac{\partial \delta}{\partial q}=0$, we observe that we have in fact that $\psi \in \operatorname{Iso}_{0}^{2}(h)$, that is $\psi$ is not only conformal, but isometric for $h$. Then, we use 1-rigidity of Riemannian metrics to deduce that $\operatorname{jet}_{0}^{2}(\psi)=1$.

- Now, $\phi \in \operatorname{Iso}_{(0,0)}^{3}(g)$ implies in particular that $\psi$ is 3 -conformal for $h$. We then apply Liouville Theorem, that is the 3-rigidity of conformal Riemannian metrics, and deduce that $\operatorname{jet}_{0}^{3}(\psi)=1$.

5.1.2. Second step: $\phi \in \mathrm{Iso}_{(0,0)}^{3}$ and $\mathrm{jet}_{0}^{3} \psi=1 \Longrightarrow \operatorname{jet}_{(0,0)}^{2} \delta=\operatorname{jet}_{(0,0)}^{2}$ r, i.e., all the second derivatives of $\delta$ vanish at $(0,0)$. The equation $\phi \in \mathrm{Iso}_{(0,0)}^{3}$ obtained by taking second derivatives of the isometric equation gives relations between $\operatorname{jet}_{0}^{2}(\delta)$ and $\operatorname{jet}_{0}^{3}(\psi)$. Since, we already know that $D_{0} \psi=1$ and all other derivatives of order $\leq 3$ vanish, we obtain equations relating second derivatives of $\delta$ (the first derivatives of $\delta$ are known). We then prove that this system of algebraic linear equations (on these derivatives) is determined and that all the second derivatives of $\delta$ vanish.

5.2. The general case, generalized conformal structures. When $g_{q, r}$ has a general form rather the split one in the transversally conformal case, we get on the quotient space a kind of generalized conformal structure. This means that at each $q \in Q$, we are giving $S_{q} \subset \operatorname{Sym}^{2 *}\left(T_{q} M\right)$, the space of Euclidean scalar products on $T_{q} Q$, such that $S_{q}$ is the image of a (non-parameterized) curve. The case of Riemannian metrics corresponds to $S_{q}$ reduced to one point, and that of conformal structures to that where all the elements of $S_{q}$ are proportional. (Of course, we assume everything depends smoothly on $q$ ).

The proof of Theorem 3.1 goes through an adaptation of Liouville theorem to generalized conformal structures, that is a generalized conformal structure is 2-rigid.

The second step in the proof of Theorem 3.1 is the same as in the transversally conformal case.

\section{Weakness}

We show in what follows how the sub-rigidity is weak in comparison to rigidity, by considering preserving structure actions of special groups (as a nice recent reference of the subject of these actions, one may consult [14]).

6.1. Gromov representation theorem for rigid structures. Let $G$ be a Lie group acting on a compact manifold $M$ by preserving an analytic geometric structure $\sigma$ and a volume form. The Gromov representation theorem concerns the case where $\sigma$ is rigid and $G$ is a simple Lie group. It states that $M$ tends to look like a quotient $G / \Lambda$, where $\Lambda$ is a co-compact lattice in $G$. The precise statement is that $\pi_{1}(M)$ is large, in the sense that it has a representation in some linear group whose the Zariski closure of the image contains a copy of the Lie group $G$ (see for instance [9]). This result was generalized for actions of lattices in $G$, by Fisher-Zimmer: 
Theorem 6.1. [15] Let $\Gamma$ be a lattice in a simple Lie group $G$ of rank $\geq 2$. Suppose $\Gamma$ acts on a manifold $M$ analytically by preserving an analytic rigid geometric structure, and ergodically for a volume form. Then, either:

(1) $\Gamma$ acts via a homomorphism in a compact subgroup $K \subset \operatorname{Diff}(M)$ (and thus $M$ is a homogeneous space $K / C$, by ergodicity), or

(2) As in Gromov representation theorem, $\pi_{1}(M)$ admits a homomorphism in some $\mathrm{GL}_{N}(\mathbb{R})$ whose Zariski closure contains a subgroup locally isomorphic to the Lie group $G$.

We will show below that this does not extend to sub-rigid structures.

Extension of Killing fields. One crucial ingredient in the proof on the previous results is that, for rigid geometric structures, local analytic Killing fields extend to the full manifold if it is simply connected (see $[1,11,16]$ ). More precisely, let $M$ be analytic, simply connected and endowed with an analytic rigid geometric structure $\sigma$. Let $V$ be a Killing field of $\sigma$ defined on an open set $U \subset M$ (that is the local flow of $V$ preserves $\sigma$ ). Then, $V$ extends (as an analytic Killing field) to $M$.

This fact is no longer true for sub-rigid structures. Indeed, let $g=x^{2 d} d x^{2}$ be a "singular" Riemannian metric on $\mathbb{R}$. On $\mathbb{R}-\{0\}$, the metric is regular, and hence flat, it has a Lie algebra of Killing fields of dimension 1. No such Killing field extends at 0 . Indeed, as 0 is the unique singular point of $g$, it will be fixed by any local isometry $\phi$ defined on its neighborhood. Furthermore there is a well defined distance $d_{g}$ derived from $g$, and thus, $d_{g}(0, x)=d_{g}(0, \phi(x))$. This implies that $\phi$ is necessarily $\pm I d$. In particular any Killing field defined around 0 vanishes in a neighborhood of 0 and hence everywhere.

6.2. No Gromov representation for sub-rigid structures. In the sub-rigid case, we have the following example:

Theorem 6.2. The lattice $\mathrm{SL}_{3}(\mathbb{Z})$ acts analytically and ergodically on a compact simply connected manifold, by preserving an analytic sub-rigid structure and a volume form. More precisely, there exists a holomorphic action of $\mathrm{SL}_{3}(\mathbb{Z}+j \mathbb{Z}), j=e^{\frac{2 \pi}{3} i}$, on a compact Calabi-Yau 3-manifold (i.e., a simply connected Kähler manifold with a holomorphic volume form). The action preserves a holomorphic sub-rigid structure, and is ergodic (it is measurably isomorphic to an affine action on a complex torus of dimension 3).

Remark 6.1. Observe this is a sub-rigid counter-example for the Fisher-Zimmer version concerning higher rank lattices actions whereas the original Gromov's theorem deals with actions of Lie groups. The suspension of a lattice action gives rise to an action of its ambient Lie group $G$, which always satisfies the Gromov representation theorem. It remains therefore open to see if an analogous result as in the theorem above is true for actions of Lie groups as $\mathrm{SL}_{3}(\mathbb{R})$ ?

Proof. Before giving the construction, let us discuss somewhat the general question of taking pull-backs of geometric structures. 
Pull-Back. Let $\pi: M^{\prime} \rightarrow M$ be a differentiable map, with $M$ and $M^{\prime}$ of same dimension $n$. Assume $M$ is endowed with an $H$-structure $\sigma\left(H\right.$ a subgroup of $\left.\mathrm{GL}_{n}(\mathbb{R})\right)$. If $\pi$ has no critical points ( $\pi$ a local diffeomorphism) then, one defines straightforwardly $\pi^{*}(\sigma)$. Indeed, $\operatorname{jet}^{1}(\pi)$ is well defined as a map $\mathrm{GL}^{(1)}\left(M^{\prime}\right) \rightarrow \mathrm{GL}^{(1)}(M)$, and then one composes it with $\sigma: \mathrm{GL}^{(1)}(M) \rightarrow \mathrm{GL}_{n}(\mathbb{R}) / H$. In contrast, there is generally no mean to define $\pi^{*}(\sigma)$ at a critical point. As an example, there is no definition of the inverse image of a vector field on $M$.

Let us now describe a situation where the definition of the pull-back of an $\mathrm{H}$ structure is possible as a geometric structure in the Gromov sense, but not as an $H$-structure. Assume $\sigma$ is a parallelism $x \rightarrow\left(e_{1}(x), \ldots, e_{n}(x)\right)$ on $M$. One defines a geometric structure $\sigma^{\prime}: \mathrm{GL}^{(1)}\left(M^{\prime}\right) \rightarrow \operatorname{Mat}_{\mathrm{n}}(\mathbb{R})$ by:

$$
u=\left(u_{1}, \ldots, u_{n}\right) \in \mathrm{GL}_{y}^{(1)}\left(M^{\prime}\right) \rightarrow \sigma^{\prime}(u)=\left(a_{i j}(u)\right) \in \operatorname{Mat}_{\mathrm{n}}(\mathbb{R}),
$$

where $D_{y} \pi\left(u_{i}\right)=\Sigma a_{i j}(u) e_{j}(\pi(y))$. In other words, $\sigma^{\prime}(u)$ is the matrix of the derivative $D_{y} \pi$ with respect to the bases $\left(u_{1}, \ldots, u_{n}\right)$ and $\left(e_{1}(\pi(y)), \ldots, e_{n}(\pi(y))\right)$ of $T_{y} M^{\prime}$ and $T_{\pi(y)} M$, respectively.

Case of the affine flat connection. Another situation which serves in the proof of our theorem is that of the usual affine structure on $\mathbb{R}^{n}$. This is an $H$-structure of order 2, i.e., a map $\sigma: \mathrm{GL}^{(2)}\left(\mathbb{R}^{n}\right) \rightarrow \mathrm{GL}_{0}^{(2)}\left(\mathbb{R}^{n}\right)$, where $\mathrm{GL}_{0}^{(2)}\left(\mathbb{R}^{n}\right)$ is the set of invertible jets in jet $_{0}^{2}\left(\mathbb{R}^{n}, \mathbb{R}^{n}\right)$ (the space of jets $\left.\left(\mathbb{R}^{n}, 0\right) \rightarrow \mathbb{R}^{n}\right)$, i.e., the inverse image of $G L_{n}(\mathbb{R})$ under the projection jet $_{0,0}^{2}\left(\mathbb{R}^{n}, \mathbb{R}^{n}\right) \rightarrow \mathrm{GL}_{n}\left(\mathbb{R}^{n}\right) \subset$ jet $_{0,0}^{1}\left(\mathbb{R}^{n}, \mathbb{R}^{n}\right)$ (the space of jets $\left.\left(\mathbb{R}^{n}, 0\right) \rightarrow\left(\mathbb{R}^{n}, 0\right)\right)$. The usual affine connection on $\mathbb{R}^{n}$ is obtained from the projection $\mathrm{GL}^{(2)}\left(\mathbb{R}^{n}\right)=\mathbb{R}^{n} \times \mathrm{GL}_{0}^{(2)}\left(\mathbb{R}^{n}\right) \rightarrow \mathrm{GL}_{0}^{(2)}\left(\mathbb{R}^{n}\right)$. Now the point is that $\sigma$ extends as a map $\bar{\sigma}: \operatorname{jet}_{0}^{2}\left(\mathbb{R}^{n}, \mathbb{R}^{n}\right) \rightarrow \operatorname{jet}_{0,0}^{2}\left(\left(\mathbb{R}^{n}, \mathbb{R}^{n}\right)\right.$. The smooth map $\pi$ always induces a map $\operatorname{jet}^{2}(\pi): \mathrm{GL}^{(2)}\left(M^{\prime}\right) \rightarrow \operatorname{jet}_{0}^{2}\left(\mathbb{R}^{n}, \mathbb{R}^{n}\right)$. We define $\pi^{*}(\sigma)$ as $\bar{\sigma} \circ \operatorname{jet}^{2}(\pi)$.

If the degeneracy of $\pi$ is bounded, that is there exists $k$ such that jet ${ }_{x}^{k}(\pi) \neq 0$, for any $x$, then $\pi^{*}(\sigma)$ is sub-rigid.

The construction is natural, and thus, if a group $\Gamma$ acts on $M^{\prime}$ and $\mathbb{R}^{n}$ equivariantly with respect to $\pi$, and if the action on $\mathbb{R}^{n}$ is affine, then, $\Gamma$ preserves the pull-back of the geometric structure on $M^{\prime}$.

All this applies identically to the torus $\mathbb{T}^{n}=\mathbb{R}^{n} / \mathbb{Z}^{n}$, since we have the same trivialization of the jet bundle $\mathrm{GL}^{(2)}\left(\mathbb{T}^{n}\right)$.

Actions. Consider $\Gamma$ a subgroup of $\mathrm{SL}_{n}(\mathbb{Z})$ and let it act as usually on $\mathbb{T}^{n}$. Blow-up a finite orbit of $\Gamma$ (e.g. a rational point), and get a manifold $M^{\prime}$ with a $\Gamma$-action. It was proved by Katok-Lewis [17] that this action is volume preserving, and by Beneviste-Fisher [4] that it preserves a sub-rigid structure, but can not preserve a rigid one.

Orbifolds. Here, we assume that there is a finite index subgroup $F \subset \mathrm{SL}_{n}(\mathbb{Z})$ commuting with $\Gamma$. We then consider the orbifold $M_{0}=\mathbb{T}^{n} / F$. It inherits a $\Gamma$-invariant natural flat affine connection in an orbifold sense.

The next step is to desingularize $M_{0}$ in order to get a (regular) manifold $M^{\prime}$ with a $\Gamma$-action equivariant with respect to a projection $M^{\prime} \rightarrow M_{0}$. 
An example. Take $F$ to be the group isomorphic to $Z / 2 \mathbb{Z}$ generated by the involution $I: x \rightarrow-x$. If $n=2$, the quotient around fixed points is just a cone with opening angle $\pi$. It follows that the so obtained orbifold is a topological surface. It is in fact a topological sphere, with exactly 4 conic singularities on which $\Gamma=\mathrm{SL}_{2}(\mathbb{Z})$ acts continuously by preserving a continuous volume form. The singularities can be solved to give the usual differentiable structure on the sphere, but this can not be done $\Gamma$-equivariantly.

Complex case. A higher dimensional generalization is possible, but in a complex framework. So, we start with a complex torus $A=\mathbb{C}^{n} / \Lambda$ (of complex dimension $n$ ). We consider $M_{0}=A / F$, where $F$ is the same previous group generated by the involution $I$. If $n \geq 2, M_{0}$ is no longer a topological manifold, since the fixed points of $I$ are not conical. We then start blowing-up $A$ on $I$-fixed points, in a complex way, and get $M_{1}$. We have a projection $p_{1}: M_{1} \rightarrow A$ with singular fibers isomorphic to $\mathbb{C} P^{n-1}$ over $I$-fixed points. Now, $F$ acts naturally on $M_{1}$ with conical singularities, and hence $M_{2}=M_{1} / F$ is a topological manifold. The resolution of singularities yields a complex structure on $M_{2}$ with a natural $\Gamma$-holomorphic action.

Our case. For $n=2$, we get a Kummer surface, a special case of K3 surfaces. Observe that the volume form $d v=d z_{1} \wedge d z_{2}$ is $F$-invariant and hence well defined on $M_{2}$. However, even if the form $p_{1}^{*}(d v)$ is singular along the exceptional fibers, it gives rise to a true regular holomorphic volume form on $M_{2}$.

In order to have a similar construction in dimension 3, we replace $F$ by the group generated by the rotation $J: z \rightarrow j z$ where $j=e^{\frac{2 \pi}{3} i}$ on $\mathbb{C}^{3}$ (see [10], Section 7.6). It preserves the volume form, and therefore, we get on the corresponding $M_{2}$ a holomorphic volume form.

Regarding the $\Gamma$-action, we take, $\Gamma=\mathrm{SL}_{3}(\mathbb{Z}+j \mathbb{Z})$ and $\Lambda=(\mathbb{Z}+j \mathbb{Z})^{3} \subset \mathbb{C}^{3}$. Thus, $\Gamma$ is a lattice in $\mathrm{SL}_{3}(\mathbb{C})$, it preserves $\Lambda$ and commutes with $J$.

As in the cases $n=1,2$, one can prove directly that $M_{2}$ is simply connected. Another idea is to use the fact that $M_{2}$ has holomorphic volume form to deduce it has a vanishing first Chern class. Then, apply Yau's theorem to get a Kähler Ricci flat metric on it. But, for such manifolds, up to a finite cover, there is a de Rham decomposition into a product of a flat torus, and (compact) simply connected manifolds (hyper-Kähler and Calabi-Yau, see [7]). Thus, it suffices to verify that $M_{2}$ has no torus (of dimension 1, 2, or 3) as a factor, to prove that it has a finite fundamental group.

Remark 6.2. One can use general theory of rigid transformation groups to see that the latter action can not preserve a (real) analytic rigid geometric structure. Indeed, by $[11,16]$, the isometry group of a unimodular analytic rigid structure on a simply connected manifold have a finite number of connected components. This means that up to a finite index, the $\Gamma$-action extends to an action of a Lie group, which can be easily seen to be impossible.

\section{Acknowledgments}

S. Bekkara was supported in part by the ANR Geodycos of the ENS-Lyon and the project RIAMI of the CIMPA. 


\section{References}

[1] A. Amores, Vector fields of a finite type G-structure, J. Differ. Geom. 14 (1979), 1-6.

[2] W. Ballmann, Geometric structures, http://people.mpim-bonn.mpg.de/hwbllmnn/notes.html Lecture notes page.

[3] Y. Benoist, Orbites des structures rigides (d'après M. Gromov). Feuilletages et systèmes intégrables (Montpellier, 1995), Progr. Math. 145, Birkhäuser, 1997, 1-17.

[4] J. Benveniste and D. Fisher, Nonexistence of invariant rigid structures and invariant almost rigid structures, Comm. Anal. Geom. 13(1) (2005), 89-111.

[5] E. Bekkara, C. Frances and A. Zeghib, Actions of semisimple Lie groups preserving a degenerate Riemannian metric, Trans. Amer. Math. Soc. 362(5), (2010), 2415-2434.

[6] S. Bekkara and A. Zeghib, On the local rigidity of degenerate Riemannian metrics, preprint 2012.

[7] A. Beauville, Variétés kähleriennes dont la première classe de Chern est nulle, J. Differ. Geom. 18 (1983), 755-782.

[8] A. Candel and Quiroga-Barranco, Parallelisms, prolongations of Lie algebras and rigid geometric structures, Manuscripta Math. 114(3) (2004), 335-350.

[9] A. Candel and R. Quiroga-Barranco, Gromov's centralizer theorem, Geom. Dedicata 100 (2003), 123-155.

[10] S. Cantat and A. Zeghib, Holomorphic actions, Kummer examples, and Zimmer program, Ann. Sci. École Norm. Sup., to appear.

[11] G. D'Ambra and M. Gromov, Lectures on transformation groups: geometry and dynamics, in 'Surveys in differential geometry', Lehigh Univ, Cambridge, MA, 1990, 19-111.

[12] S. Dumitrescu, Meromorphic almost rigid geometric structures, 'Geometry, rigidity, and group actions', 3258, Chicago Lectures in Math., Univ. Chicago Press, Chicago, IL, 2011.

[13] C. Fefferman and R. Graham, Conformal invariants, in 'The mathematical heritage of Élie Cartan', Lyon, 1984, Astérisque 1985, Numero Hors Série, 95-116.

[14] D. Fisher, Groups acting on manifolds: around the Zimmer program, in 'Geometry, rigidity, and group actions', Chicago Lectures in Math., Univ. Chicago Press, Chicago, IL, 2011, 72-157.

[15] D. Fisher and R. Zimmer, Geometric lattice actions, entropy and fundamental groups, Comment. Math. Helv. 77(2) (2002), 326-338.

[16] M. Gromov, Rigid transformations groups, Géométrie différentielle (Paris, 1986), 65-139, Travaux en Cours, 33, Hermann, Paris, (1988).

[17] A. Katok and J. Lewis, Global rigidity results for lattice actions on tori and new examples of volume-preserving actions, Israel J. Math. 93 (1996), 253-280

[18] S. Kobayashi, Transformation groups in differential geometry, Springer-Verlag, (1972).

Ust-Oran, Algeria

E-mail address: samir.bekkara@gmail.com

CNRs, Umpa, Ens-Lyon, France

E-mail address: abdelghani.zeghib@ens-lyon.fr

http://www . umpa.ens-lyon.fr/ $\sim_{\text {zeghib/ }}$ 